ФТИ-2020

\title{
PARTICULATE MATTER VARIATION FOR DIFFERENT TYPES OF CIGARETTES IN INDOOR AIR
}

\author{
Khalaf H.N. ${ }^{1,3}$, Mostafa M.Y.A. ${ }^{1,3}$, Zhukovsky M.V. ${ }^{1,2}$ \\ 1) Ural Federal University, Yekaterinburg 620002, Russia \\ ${ }^{2)}$ Institute of Industrial Ecology, Ekaterinburg, Russia \\ 3) Minia University, Faculty of Science, Department of Physics, El-Minia, Egypt \\ E-mail: hyamnazmy@mail.ru
}

The present study aims to characterize PM emissions from three different types of cigarettes (Electronic cigarettes, tobacco cigarettes and IQOS cigarettes). The experiments were carried out in a $65 \mathrm{~m} 3$ lab room with burning the three types of cigarettes separately. PM1, PM2.5, PM10 concentrations.

Particulate matter inhalation (PM) in second hand smoke is harmful to smokers and non-smokers. Various additions to cigarettes may affect the amount from PM. Exposure to PM is not only caused lung cancer but also increased risk of stroke breast cancer mortality. Although the main risks may be transferred to the distal lung by particulate matter, A few studies have been interested in studying PM. The present study aims to characterize PM emissions from three different types of cigarettes (Electronic cigarettes, tobacco cigarettes and IQOS cigarettes). The experiments were carried out in a $65 \mathrm{~m}^{3}$ lab room with burning the three types of cigarettes separately. PM1, PM2.5 and PM10 concentrations were measured simultaneously with aerosol diffusion spectrometer (ADS) 1. The Electronic cigarettes achieved the highest values of particulate matter with $193,1 * 10^{3}$ and $2 * 10^{3}\left(\mu \mathrm{g} / \mathrm{m}^{3}\right)$ for PM1, PM2.5 and PM10 respectively. The value of surface area ranged from 3460 for IQOS cigarettes to 7482 for Electronic cigarettes. IQOS cigarettes got less particulate matter concentrations in different sizes. This may be due to the way IQOS cigarettes operate, as the tobacco is heated, not burned. Time dependant of PM10 and surface area concentration for the three types of cigarettes are shown in figure 1.
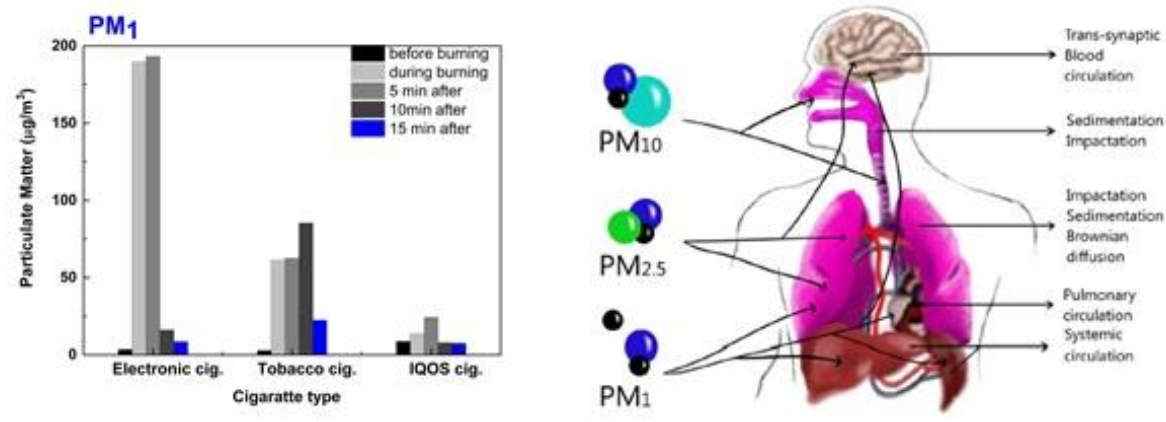

Fig. 1. Time dependant of PM10 and surface area concentration for the three types of cigarettes.

1. Khalaf H.N., Mostafa M.Y.A., Vasyanovich M., Zhukovsky M. Applied Radiation and Isotopes 145, 95-100. 